\title{
Peripheral neuropathy with ethambutol
}

\author{
P. TUGWELL* \\ M.B., M.R.C.P. \\ Whittington Hospital, N.19
}

\author{
S. L. JAMES \\ B.Sc., M.B., B.S., D.T.M. \& H. \\ Royal Northern Hospital, N.7
}

\begin{abstract}
Summary
Three cases are described in which peripheral neuropathy occurred while the patients were taking ethambutol. The neuropathy regressed when the drug was stopped.
\end{abstract}

\section{Introduction}

Ethambutol is a promising antituberculous drug which has been proposed as a replacement for PAS in the first-line triple therapy of tuberculosis. This suggestion is based on its equal or possible superior effectiveness in man, compared with PAS (Donomae \& Yamamoto, 1966; Bobrowitz, 1966), whilst having few toxic effects in the doses at present recommended (Tubercle, 1966; Tiburtius, 1970).

Ethambutol is well-documented as the cause of optic neuritis (Leibold, 1966) but an extensive search of the literature has revealed no previous published case reports of peripheral neuropathy. The following three instances of peripheral neuropathy, associated with ethambutol therapy, are presented as further evidence of its neurotoxicity.

\section{Case 1}

A 52-year-old Iranian presented in April 1971, with poor vision, poor hearing, pain and numbness of the legs with difficulty in walking, and numbness and clumsiness of the hands.

Two and a half years previously he had developed fever, night sweats and cough and had lost $2 \frac{1}{2}$ stone in weight. Pulmonary tuberculosis was eventually diagnosed and he was admitted to a sanatorium where he was treated with streptomycin, isoniazid and ethambutol.

He received streptomycin $1 \mathrm{~g}$ thrice weekly for 7 months; isoniazid $300 \mathrm{mg}$ daily for 4 months, then $200 \mathrm{mg}$ daily; and ethambutol $2500 \mathrm{mg}$ daily for 3 months $(50 \mathrm{mg} / \mathrm{kg})$, then $2000 \mathrm{mg}$ daily for 3 months ( $40 \mathrm{mg} / \mathrm{kg})$ and then $1500 \mathrm{mg}$ daily $(30 \mathrm{mg} /$ $\mathrm{kg}$ ). On this antituberculous therapy his general condition improved and he regained $1 \frac{1}{2}$ stone in weight.

Nine months prior to admission to our hospital ( 9 months after starting therapy) he began to get pain in his legs. This pain extended from knee to

\footnotetext{
* Present address: Ahmadu Bello University Hospital, Zaria, Nigeria.
}

foot and was associated with numbness and inability to move his toes. He developed increasing difficulty in walking. A short time afterwards he noticed difficulty in fine movements of his hands and he became unable to lift or hold things.

His isoniazid was stopped as it was felt that his symptoms could be due to isoniazid neuropathy. In addition, he was treated with vitamins $B_{1}, B_{2}$, $B_{12}$ and $B_{6}$ by mouth and by injection, but his condition did not improve. He continued to take ethambutol $1500 \mathrm{mg}$ daily.

Six months prior to admission his sight began to deteriorate until with his right eye he was unable to read even large print, see television or make out any detail of faces or objects; while with his left eye he was unable to tell light from dark. At the same time as his vision began to fail, his hearing became mildly impaired in the left ear and he had occasional tinnitus.

On examination he was thin with marked wasting of the limb muscles. His gait was slow, shuffling and unsteady, even with the aid of two sticks. Examination of the eyes: normal pupillary reactions in both eyes, fundi showed bilateral optic atrophy. In the right eye there was tubular vision to large objects only, and in the left eye there was no vision.

Neurological examination of the arms revealed impairment of pin-prick, temperature and light touch affecting both surfaces of the hands. Twopoint discrimination was absent in thumbs and index fingers. Vibration sense was diminished in the arms but postural sense was normal. Biceps and triceps jerks were present and equal but right supinator reflex was absent.

In the lower limbs there was impairment of pinprick, temperature and light touch below the knees. In addition, there was severe impairment of vibration sense at the knees and complete absence of vibration sense at the ankles. Postural sense was absent in both big toes. There was bilateral muscle wasting and severe tenderness in the muscles of both legs. The knee jerks were just present and the ankle jerks were absent. The plantar responses were flexor. The motor power of the legs showed marked impairment: he was unable to move his toes at all and only just able to move his ankles.

Physical examination of the lungs showed some 
reduction in air entry in the right upper zone. Examination of the abdomen revealed a large cystic mass in the left loin. Vestibular and auditory testing showed no vestibular abnormality, but there was a high tone sensory neural hearing loss.

Chest X-ray showed ill-defined fibrotic shadows with some nodules of calcification in the right mid and upper zones. X-ray of the spine showed wedgedeformity of the twelfth thoracic vertebral body with appearances suggestive of old trauma.

In the X-ray of the abdomen, the psoas muscle outline and the kidney could not be defined on the left side, and there was absence of bowel gas shadowing in this region. IVP showed normal kidneys, and a mass below the lower pole of the left kidney with lateral displacement of the left ureter.

The abdominal mass was thought to be a retroperitoneal cyst and laparotomy confirmed this. Histological appearance was consistent with this being massive fat necrosis in an old encysted haematoma, probably of traumatic origin.

Urine and sputum were negative for acid-fast bacilli on many occasions and his tuberculosis was considered to be inactive. Ethambutol, the only antituberculous drug he was still taking, was then stopped. His condition improved in all respects. His leg pains diminished, sensation improved and with the aid of physiotherapy he was able to walk well after 2 months. A month later he was able to read well with his right eye and was beginning to see shapes and outlines with his left eye. The impairment of hearing ceased to be noticeable to the patient and he had no more tinnitus.

The ocular toxic effects in the patient were undoubtedly due to ethambutol, and there seems little doubt that the severe peripheral neuropathy was also due to this drug. There was no improvement when isoniazid was discontinued and it was not until the ethambutol was stopped some months later that the relentless progress of the neuropathy was halted and improvement began. It is difficult to decide whether the impairment of hearing in this case was the result of streptomycin or ethambutol, although the fact that it did not start until 5 months after streptomycin was stopped indicates that ethambutol was the likely cause.

\section{Case 2}

This 50-year-old housewife with chronic airways obstruction was admitted to hospital in November 1970 , where she was diagnosed as having pulmonary tuberculosis. Acid-fast bacilli were present on direct smear of the sputum, and numerous cavities were present on chest X-ray. She was also found to have a megaloblastic anaemia which failed to respond to vitamin $B_{12}$, but the reticulocyte count and haemoglobin level rose following folate orally. This anaemia was considered to be dietary in origin. She had a normal Schilling test and free acid was present in the stomach, excluding pernicious anaemia.

Her tuberculosis was treated with streptomycin, isoniazid and PAS. However, she developed dizziness which was ascribed to streptomycin, and confusion and paranoia ascribed to isoniazid. Therefore she was started on rifampicin $600 \mathrm{mg}$ daily and ethambutol $400 \mathrm{mg}$ b.d. $(18.5 \mathrm{mg} / \mathrm{kg})$ together with prednisolone in view of her poor general condition. She was also given amitriptyline $10 \mathrm{mg}$ t.d.s. for depression, and was sedated with chlorpromazine $25 \mathrm{mg}$ t.d.s. She made slow progress, but her sputum eventually became negative on direct smear, and her condition improved sufficiently for her to be discharged from hospital in April 1971.

She was re-admitted in May 1971, complaining of numbness and tingling in the fingers, together with a definite clumsiness of the small movements of the hand. She had also noted a rash over the front of both shins for the previous fortnight. At this time she was still taking the following drugs: rifampicin $600 \mathrm{mg}$ daily, ethambutol $400 \mathrm{mg}$ b.d., amitriptyline $10 \mathrm{mg}$ t.d.s., chlorpromazine $25 \mathrm{mg}$ t.d.s., prednisolone $5 \mathrm{mg}$ b.d., folic acid $5 \mathrm{mg}$ b.d., vitamin $B_{12} 250 \mu \mathrm{g}$ i.m. monthly.

Physical examination revealed a purpuric rash on both shins which was not perifollicular. In the nervous system there was no evidence of motor weakness, but pin-prick and temperature sensation were diminished distally in the arms, and vibration sense was absent up to the anterior iliac spines in the lower limbs. The skin over the soles of her feet was extremely tender. Other sensory modalities were normal. Tendon reflexes were diminished, but present with reinforcement, and both plantar responses were flexor.

Investigations: Platelets $216,000 / \mathrm{mm}^{3}$. Normal prothrombin and partial thromboplastin time. Haemoglobin $13.2 \mathrm{~g} / 100 \mathrm{ml}$. Serum $B_{12} 340 \mathrm{pg} / \mathrm{ml}$. Serum folate $30 \mathrm{ng} / \mathrm{ml}$. Electromyography showed no abnormality on muscle sampling with a concentric needle electrode. Motor conduction velocity was diminished in the left median nerve $(44 \mathrm{~m} / \mathrm{sec})$ and in the left lateral popliteal nerve $(40 \mathrm{~m} / \mathrm{sec})$. Sensory conduction was absent between the index finger and wrist in the left median nerve. Glucose tolerance test was normal. Urinary porphyrins negative. Pyruvate tolerance test normal $(0.7 \mathrm{mg} / 100 \mathrm{ml}$ at $90 \mathrm{~min})$.

All the drugs that she had been taking were discontinued apart from the prednisolone, but in view of the active tuberculosis she was then given capreomycin and prothionamide whilst the steroids were tailed off. The purpura and paraesthesiae disappeared and the numbness improved somewhat over the next few weeks. Rifampicin was not 
considered to be responsible for the purpura or neuropathy and was therefore restarted 2 weeks later instead of capreomycin. She has continued on prothionamide and rifampicin, so far for 3 months without recurrence of the purpura or paraesthesiae. There is still some residual numbness. The cause of the purpura was not defined, although it may have been due to the anti-anabolic action of prednisolone.

The neuropathy was not thought to be associated with her megaloblastic anaemia, since the electromyography showed no evidence of cord damage, and the serum $B_{12}$ and folate and haemoglobin were normal on her second admission. Although the patient was known to have had a reasonably high ethanol intake in the past, her intake between the two admissions had, according to the social workers, been less than her usual quantity. Her normal pyruvate tolerance test makes it unlikely that ethanol was the cause of her neuropathy. It was therefore considered likely that the neuropathy was due to a drug which she was receiving.

Peripheral neurpathy is not a recognised complication of therapy with rifampicin, chlorpromazine, folic acid or prednisolone. A very few cases have been reported with amitriptyline, but all were receiving a dose of more than $50 \mathrm{mg}$ t.d.s. while our patient was only receiving $10 \mathrm{mg}$ t.d.s. (Isaacs \& Carlish, 1963). It was therefore felt that the most likely cause was ethambutol.

\section{Case 3}

This 50-year-old sales clerk was admitted to hospital in February 1971, with pulmonary tuberculosis and a deep vein thrombosis in the right leg. Examination of the nervous system was normal apart from a fixed, dilated left pupil, presumably due to an episode of iritis in the past. Chest X-ray showed widespread shadowing with cavities in both upper lobes. Acid-fast bacilli were seen on direct smear of the sputum, and on culture were fully sensitive to streptomycin, isoniazid and PAS.

He was not treated with anticoagulants in view of a past history of melaena from a duodenal ulcer. Antituberculous therapy was started with streptomycin, isoniazid and PAS but drugs were discontinued after 3 weeks when the patient developed a pyrexia and tachycardia. Treatment was restarted with isoniazid $150 \mathrm{mg}$ b.d., PAS $6 \mathrm{~g}$ b.d. and ethambutol $400 \mathrm{mg}$ b.d. $(13 \mathrm{mg} / \mathrm{kg})$. He made a good clinical improvement and his sputum on direct smear became negative for acid-fast bacilli. He was discharged in April 1971.

He was seen again in July 1971, when he complained of tingling in the fingers of 3 weeks' duration. Physical examination showed no objective neurological signs. The chest $\mathrm{X}$-ray showed continuing clearing of shadowing in the lung fields. Electro- myography showed no abnormality on muscle sampling with a concentric needle electrode. Motor conduction tests showed no reduction of motor conduction velocity in right median and right ulnar nerve. Sensory conduction tests showed reduction in amplitude and some delay in sensory potentialsright median nerve, index finger to wrist: $4 \mu \mathrm{V}$; $3.5 \mathrm{msec}$, right ulnar nerve, little finger to wrist: $5 \mu \mathrm{V}, 3.25 \mathrm{msec}$. These findings are consistent with a mild sensory neuropathy.

The ethambutol was stopped whilst the isoniazid and PAS were continued and rifampicin $600 \mathrm{mg}$ was added. His symptoms regressed spontaneously over the following weeks.

Peripheral neuropathy was unlikely to be due to the isoniazid as he was receiving a small dose $(5 \mathrm{mg} / \mathrm{kg})$ divided into a twice-daily dosage, at which level peripheral neuropathy is extremely rare (Tuberculosis Chemotherapy Centre, Madras, 1963) and his symptoms resolved whilst continuing to take the same dose of isoniazid. It therefore seems that ethambutol was a likely cause of this man's peripheral neuropathy.

\section{Discussion}

Peripheral neuropathy is a little-recognized complication of ethambutol therapy. There have been no previous cases reported to the Committee on Safety of Drugs in Britain.

In an unpublished series collected by Lederle Laboratories (Lederle, personal communication, 1971) of 1024 patients treated with ethambutol, fifteen suffered from peripheral neuritis, mainly in the form of numbness of the extremities, at some time during the therapy. Of these fifteen, only four were receiving ethambutol alone; concomitant drugs were isoniazid, ethionamide, capreomycin, PAS, kanamycin, isovincamine. Doses of ethambutol in patients experiencing this side-effect ranged from $10-19 \mathrm{mg} / \mathrm{kg}$ on alternate days, to $40 \mathrm{mg} / \mathrm{kg}$ daily.

There have been some reports (Donomae \& Yamamoto, 1966; American Council of Drugs, 1969; Wilson, 1970) of peripheral neuropathy with numbness and tingling of the extremities due to ethambutol, but no case reports are given. The third of these reports referred to the unpublished findings of Lederle Laboratories mentioned above (Wilson, 1971). Schmidt (1966) found that, in monkeys, high doses of ethambutol caused severe neurotoxic effects resulting in unsteady gait, loss of equilibrium and other disturbances in co-ordination. However, in this series only the pathological changes in the central nervous system were studied, so how much of the clinical picture was due to peripheral neuropathy is not known.

The neuropathy in our case was mainly sensory, although there was evidence of motor deficit in 
Case 1 and 2. It should be noted that this side-effect was found in the two patients receiving low doses of ethambutol (less than $20 \mathrm{mg} / \mathrm{kg}$ daily) as well as in the patient receiving much higher dosage $(50 \mathrm{mg} / \mathrm{kg}$ daily).

It is interesting to compare the peripheral neuropathy with the optic neuritis associated with ethambutol. The finding of peripheral neuropathy, together with optic neuritis in Case 1 suggests a possible susceptibility of certain persons to the neurotoxic effects of ethambutol. The time of onset of these two complications is similar-our patients developed symptoms after 5-9 months' treatment which is similar to the average time of onset of the optic neuritis (Tubercle, 1966). Our cases have shown good evidence of recovery, which again is like the optic neuritis.

It has been suggested that the pathogenesis of the optic neuritis is due to ethambutol binding zinc into its -NH groups (Kanther, 1970). The zinc is then not available for its vital function as a co-factor for various important enzymes, such as lactic acid dehydrogenase, alcohol dehydrogenase and possibly others involved in neural metabolism. It has been shown in dogs that the zinc content of the retina is reduced by administration of ethambutol, and that the drug also lowers total body stores (Buyske, Sterling \& Peets, 1966). This hypothesis is consistent with the reversibility of ocular effects on stopping the drug and would equally well apply to peripheral neuropathy.

It is important to recognize the complication of peripheral neuropathy in patients receiving ethambutol, especially when they are also receiving isoniazid, lest the latter drug alone be stopped with progression of the neuropathy.
Acknowledgments

We would like to thank Dr P. Davies and Dr C. W. H. Havard for their permisison to publish these case reports and for their help in writing this paper.

\section{References}

American Council on Drugs (1969) Evaluation of a new antituberculous agent, ethambutol hydrochloride. Journal of the American Medical Association, 208, 2463.

Bobrowitz, I.D. (1966) Comparison of ethambutol-INH versus INH-PAS in original treatment of pulmonary tuberculosis. Annals of the New York Acadamy of Sciences, 135, 921.

Buyske, D.A., Sterling, W. \& Peets, E. (1966) Pharmacological and biochemical studies on ethambutol in laboratory animals. Annals of the New York Academy of Sciences, 135, 711.

Donomae, I. \& Yамамото, K. (1966) Clinical evaluation of ethambutol in pulmonary tuberculosis. Annals of the New York Academy of Sciences, 135, 85.

IsAACS, A.D. \& CARLISH, S. (1963) Peripheral neuropathy after amitriptyline. British Medical Journal, 1, 1839.

KANTHER, R. (1970) Discussion on ethambutol. Antibiotica et Chemotherapia, 16, 311.

LEIBOLD, J.E. (1966) The ocular toxicity of ethambutol and its relation to dose. Annals of the New York Academy of Sciences, 135, 904.

SchmidT, I.G. (1966) Central nervous system effects of ethambutol in monkeys. Annals of the New York Academo of Sciences, 135, 759.

Tiburtius, H. (1970) The undesired side-effects of myam butol. Antibiotica et Chemotherapia, 16, 298.

TuberCle (1966) Leading Article. 47, 292.

Tuberculosis Chemotherapy Centre, Madras (1963) The prevention and treatment of promiazid toxicity in the therapy of pulmonary tuberculosis: 2. An assessment of the prophylactic effect of pyridoxine in low dosage. Bulletin of the World Health Organization, 29, 457.

Wilson, T. M. (1970) Clinical experience with ethambutol. Antibiotica et Chemotherapia, 16, 222.

WrLson, T.M. (1971) Personal communication. 